\title{
Discrete Curvature and Torsion-based Parameterization Scheme for Data Points
}

\author{
Xiongbing Fang ${ }^{1}$ \\ China Ship Development and Design Center \\ Wuhan, 430064, China \\ E-mail: fangxb2013@sina.cn \\ Sheng Wu \\ China Ship Development and Design Center \\ Wuhan, 430064, China \\ E-mail:wusheng7851@aliyun.com
}

\section{Jiangyuan Liu}

China Ship Development and Design Center

Wuhan, 430064, China

E-mail: liu_jiangyuan@tom.com

Parameterization for data points is a fundamental problem in the field of Computer Aided Geometric Design. Recently, an improved centripetal parameterization technique is proposed by Fang et al and its superiority to other common methods, such as uniform method, chord length method, centripetal method, Foley method and universal method, etc., is validated by a lot of numerical examples. In this article, a refined formula is firstly given to resolve the problem that the parameter values obtained by Fang's method are usually a little bigger than the optimum ones. Furthermore, as enlightened by the local differential geometric properties of parametric curves, a novel parameterization scheme is put forward by introducing the discrete curvature and torsion information at each point. As indicated by numerical experiments, the deviation measured by a curvature and Euler distance-based criterion between the interpolation B-spline curve obtained by our method and the polyline constructed by the points are smaller than the ones between the curves obtained by Fang's method, the chord length method, the standard centripetal method and the polyline. The proposed algorithm is applicable to both 2D and 3D points and has a distinct advantage for $3 \mathrm{D}$ points as compared with the three aforementioned methods.

\section{CENet2017}

22-23 July 2017

Shanghai, China

${ }^{1}$ Speaker and Corresponding author 


\section{Introduction}

Parameterization for a set of data points is one of the fundamental problems in curve and surface interpolation applications[1-3]. Appropriate parameters of data points can be further used to construct a knot vector and compute the control points of the interpolation curve. The common parameterization methods are uniform[1], chord length[4], centripetal[5,1], Foley[6] and universal methods[7,8], among which the chord length and centripetal methods are the most popular parameterization algorithm in the CAGD community. Recently, a refined centripetal parameterization method is presented by Fang et al[9]. All the aforementioned methods are obtained by mainly taking planar points as research focus and utilizing the information of distance between any two successive points, the angle between two successive lines and the discrete curvature at each point, and they are usually used in 3D points' parameterization and curve/surface interpolation problems directly.

The parameterization is also one of the key steps for 3D data points and surface interpolation[2,8]. At present, the familiar way is directly applying the above methods in 3D point cases. For spacing points, there's usually an angle $\varphi_{i}$ between the triangles constructed by the three consecutive points $\boldsymbol{P}_{i-1}, \boldsymbol{P}_{i}, \boldsymbol{P}_{i+1}$ and $\boldsymbol{P}_{i}, \boldsymbol{P}_{i+1}, \boldsymbol{P}_{i+2}$ (see Fig. 1). It is evident that the chord length and centripetal methods only utilize the distance information. Furthermore, Fang used the information of the external angle $\theta_{i}$ rather than the angle $\varphi_{i}$. When the value of $\varphi_{i}$ is not equal to zero, the interpolation curve is not only crooked but also torsional at the point $\boldsymbol{P}_{i+1}$. From the differential geometry point of view, the local configuration of a space curves at a point is confirmed by both of the curvature and torsion[10]. In this paper, we concentrate the problem of 3D points' parameterization and a new parameterization scheme fully distilled the high-order differential geometric quantities, such as discrete curvature and torsion, implied by the points is put forward, which is also appropriate for 2D points.

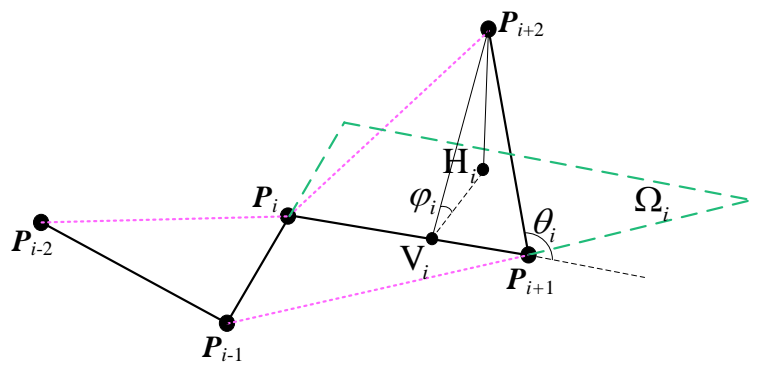

Figure 1: Spatial Structure of 3D Points

\section{Data points parameterization}

\subsection{B-spline curves}

B-spline has been proven to be the most popular representation method for free-form curves and surfaces. In the paper, we adopt B-spline curve interpolation to illuminate our parameterization scheme. A $k^{\text {th }}$-order B-spline curve is represented as

$$
\boldsymbol{P}(t)=\sum_{j=0}^{n} \boldsymbol{B}_{j} N_{j, k}(t)
$$

where $\left\{\boldsymbol{B}_{j}\right\}$ is the control points, and $\left\{N_{j, k}(t)\right\}$ is the normalized kth-order B-spline basis functions defined on the knot vector $\boldsymbol{T}=\left\{\tau_{j}\right\} j=0,1, \ldots, n+k$. The B-spline curve interpolation problem is formulated as follows. Given the points $\left\{\boldsymbol{P}_{i}\right\} i=n+1$, the associating parameter values $t_{i}$ for each point $\boldsymbol{P}_{i}$ can be obtained by a parameterization method and the knot vector $\boldsymbol{T}$ can be computed by the average method [1] as follows: 


$$
\begin{aligned}
& \tau_{0}=\cdots=\tau_{k} \\
& \tau_{m-k}=\cdots=\tau_{m} \\
& \tau_{i}=\frac{1}{k} \sum_{i=j}^{j+k+1} t_{i}, \quad j=1,2, \cdots, n-k .
\end{aligned}
$$

Then the control points $\left\{\boldsymbol{B}_{j}\right\}$ of a B-spline curve are unknowns to be calculated to satisfy $\boldsymbol{P}\left(t_{i}\right)=\boldsymbol{P}_{i}, i=0,1, \ldots, n$, i.e. the interpolation curve $\boldsymbol{P}(t)$ passing through the points at the associated parameter values.

\subsection{Common parameterization methods}

There are several classical techniques to choose the parameters $\left\{t_{i}\right\}$, such as uniform, chord length, centripetal, Foley and universal method, etc.. The uniform method does not use the distance information between each pair of consecutive points, while the chord length and centripetal ones utilize the information. The uniform, chord length and centripetal methods can be formulated uniformly as follows.

Let $\boldsymbol{L}_{i}=\boldsymbol{P}_{i}-\boldsymbol{P}_{i-1}, i=1, \ldots, n, D=\sum_{i=1}^{n}\left\|\boldsymbol{L}_{i}\right\|^{a}, t_{0}=0, \Delta t_{i}=t_{i}-t_{i-1}$, then the three methods can be rewritten as

$$
\Delta t_{i}=\left\|\boldsymbol{L}_{i}\right\|^{a} / D
$$

Equation (2.1) denotes the uniform, chord length and centripetal parameterization when $a=0, a=1$ and $a=1 / 2$, respectively. The Foley parameterization modifies the centripetal method by adopting a Nielson metric to define the distance. It is formulated as

$$
\begin{aligned}
& \Delta t_{0}=t_{1}-t_{0}=d_{0}\left[1+\frac{3 \hat{\theta}_{1} d_{1}}{2\left(d_{0}+d_{1}\right)}\right], \\
& \Delta t_{n-1}=t_{n}-t_{n-1}=d_{n-1}\left[1+\frac{3 \hat{\theta}_{n-1} d_{n-2}}{2\left(d_{n-2}+d_{n-1}\right)}\right], \\
& \Delta t_{i}=t_{i}-t_{i-1}=d_{0}\left[1+\frac{3 \hat{\theta}_{i} d_{i-1}}{2\left(d_{i-1}+d_{i}\right)}+\frac{3 \hat{\theta}_{i+1} d_{i+1}}{2\left(d_{i}+d_{i+1}\right)}\right],
\end{aligned}
$$

For more details about the definition of $d_{i}$, please refer to [6].

Lim develops a new parameterization approach named universal based on the above methods[7,8].The key idea of this method is using the parameter $\bar{t}_{i}$ associated with the maximum of the basis function $N_{j, k}(t)$.

Among the aforementioned methods, Foley and the universal parameterization are not very popular because of their laborious computation. The uniform method is rarely used as its bad parameterization effect for the final interpolation curves. So the popular parameterization methods in the context of CAGD are chord length and centripetal algorithms.

\subsection{Fang's parameterization method}

Fang et al provided a refinement of the classical centripetal parameterization method by introducing the discrete curvature information at each point[9]. The geometric intuition of the method is that the flexure of a curve at one point will elongate the arc length around the neighborhood of the point. For the points' parameterization, it signifies the parameter interval between the point and the next one will be augmented. Fang's method is formulated as

$$
\Delta t_{i}=\left\|\boldsymbol{L}_{i}\right\|^{0.5} / D+e_{i}
$$




$$
e_{i}=\alpha\left(\frac{\theta_{i} l_{i}}{2 \sin \left(\theta_{i} / 2\right)}+\frac{\theta_{i-1} l_{i-1}}{2 \sin \left(\theta_{i-1} / 2\right)}\right)
$$

where $\theta_{i}$ and $e_{i}$ are the exterior angle of the triangle $\Delta \boldsymbol{P}_{i-1} \boldsymbol{P}_{i} \boldsymbol{P}_{i+1}$ and the parameter disturbance quantity at point $\boldsymbol{P}_{i}$ respectively, the coefficient $\alpha$ is determined as 0.1 by an experimental way, and $l_{i}$ is the magnitude of the shortest side of the triangle $\Delta \boldsymbol{P}_{i-1} \boldsymbol{P}_{i} \boldsymbol{P}_{i+1}$.

The characteristics of Fang's method are summarized as follows: (1) it has fully used the geometric information of distance, angle and discrete curvature of the given point set; (2) as compared with the centripetal and other methods, the final interpolation curve obtained by Fang's has less deviation to the polyline constructed by the points and the parameters for the points are more optimized. Moreover, its computation is also compact.

\section{Curvature and torsion-based parameterization}

\subsection{Refinement of Fang's method [9]}

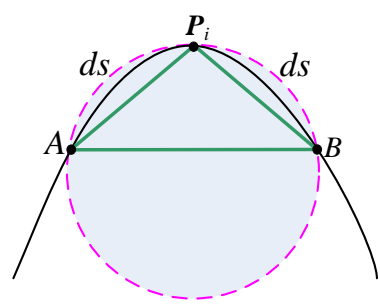

Figure 2: Discrete Curvature Circle at Point $\boldsymbol{P}_{i}$

In Fig. 2, Fang's method used the circular arc $\boldsymbol{P}_{i} \boldsymbol{B}$ of the circumcircle of the triangle $\triangle \boldsymbol{A} \boldsymbol{P}_{i} \boldsymbol{B}$ to approximate the arc length increment $d s$ of the curve from $\boldsymbol{P}_{i}$ to $\boldsymbol{B}$. Obviously, when use the circumcircle of the triangle $\Delta \boldsymbol{A P}_{i} \boldsymbol{B}$ to approximate the local of the curve around $\boldsymbol{P}_{i}$, the local of the curve is geometrically inside the circle and that means the arc length of $\boldsymbol{P}_{i} \boldsymbol{B}$ is bigger than $d s$.

Based on the above observation and the simulation results of the method, we've found that the disturbance quantity $e_{i}$ in Fang's article is bigger than its optimum. Aiming at the limitation, we give a corrected way by using the average of the chord length $\boldsymbol{P}_{i} \boldsymbol{B}$ and the arc length $\boldsymbol{P}_{i} \boldsymbol{B}$ to approximate the increment $d s$, i.e. $d s=\left(\left\|\boldsymbol{P}_{i} \boldsymbol{B}\right\|+\theta_{i} r_{i}\right) / 2$.

Suppose $\left\|\boldsymbol{P}_{i} \boldsymbol{B}\right\|=l_{i}$, the new disturbance quantity is $\bar{e}_{i}=\alpha \times d s$ instead of $e_{i}$ in Equation (2.3), then one has

$$
\bar{e}_{i}=\alpha\left(\frac{l_{i}+\theta_{i} r_{i}}{2}+\frac{l_{i-1}+\theta_{i-1} r_{i-1}}{2}\right)
$$

where $l_{i}$ is the length of the shortest side of the triangle $\Delta \boldsymbol{P}_{i-1} \boldsymbol{P}_{i} \boldsymbol{P}_{i+1}, r_{i}=l_{i} / 2 \sin \varphi_{i}$ and $\varphi_{i}=\theta_{i} / 2$. Substitute them into Equation (3.1) then it follows that:

$$
\bar{e}_{i}=\left\{\begin{array}{l}
\frac{1}{2} \alpha\left[l_{i}\left(1+\frac{\varphi_{i}}{\sin \varphi_{i}}\right)\right], i=1 \\
\frac{1}{2} \alpha\left[l_{i}\left(1+\frac{\varphi_{i}}{\sin \varphi_{i}}\right)+l_{i-1}\left(1+\frac{\varphi_{i-1}}{\sin \varphi_{i-1}}\right)\right], \quad i=2, \cdots, n-1 \\
\frac{1}{2} \alpha\left[l_{i-1}\left(1+\frac{\varphi_{i-1}}{\sin \varphi_{i-1}}\right)\right], i=n
\end{array}\right.
$$

Thereby the parameter value for each point $\boldsymbol{P}_{i}(i=1, \ldots, n)$ in the given data set can be computed 


$$
t_{i}=t_{i-1}+\left\|\boldsymbol{L}_{i}\right\|^{0.5} / D+\bar{e}_{i}
$$

where $t_{0}=0$.

\subsection{Analysis for the two methods}

From Fig. 3, we can get the following conclusions by analyzing the formulations of Equation (2.3) and (3.1).

(1) If $\varphi_{i} \in[0, \pi / 2]$, then $\frac{\varphi_{i}}{\sin \varphi_{i}} \in[1,1.57], \frac{\varphi_{i}+\sin \varphi_{i}}{2 \sin \varphi_{i}} \in[1,1.235]$. It shows that the range of the value of Equation (4) is smaller than that of Fang's.

(2) If $\varphi_{i}$ increases, then $\frac{\varphi_{i}}{\sin \varphi_{i}}$ and $\frac{\varphi_{i}+\sin \varphi_{i}}{2 \sin \varphi_{i}}$ also increase, but the change rate of the latter is more slowly than that of the anterior one. For a same $\varphi_{i}$, the quantity computed by Equation (3.1) is smaller than that of Equation (2.3).

Consequently, the parameter correction $\bar{e}_{i}$ computed by Equation (3.2) is smaller than that of Fang's.

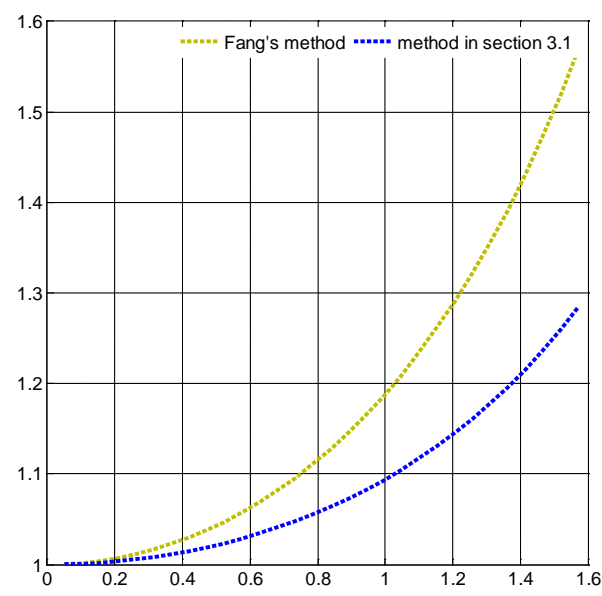

Figure 3: Comparison of the Proposed Method and Fang’s Method

\subsection{Discrete curvature and torsion-based method}

Given a set of 3D points randomly, the fitting curves usually have torsion as the points are not coplanar. The common parameterization methods like chord length and centripetal, and Fang's method usually take the geometric information of point position, lengths of line segments, angles between the line segments and the discrete curvature at each point into account rather than the higher-order geometric information of torsion. According to the local differential geometric characteristics of a space curve, the shape of the curve around a point is only determined by the curvature and torsion at the point. Based on the method in Section 3.1, a discrete curvature and torsion-based parameterization scheme is proposed for 3D points.

There are a lot of methods to calculate the discrete curvature and torsion[11-15]. In the paper, we choose the following approach to estimate the torsion at each point $[14,15]$. Let $\Delta_{i}=\left[\boldsymbol{L}_{i}\right.$, $\left.\boldsymbol{L}_{\mathrm{i}+1}, \boldsymbol{L}_{i+2}\right], i=2, \ldots, n-2$, and $[\boldsymbol{U}, \boldsymbol{V}, \boldsymbol{W}]$ be the scalar triple product of the vectors $\boldsymbol{U}, \boldsymbol{V}$ and $\boldsymbol{W}$, then one has

$$
\hat{\tau}_{i+1}=\frac{4}{\left\|\boldsymbol{L}_{i} \otimes \boldsymbol{L}_{i+1}\right\|\left\|\boldsymbol{L}_{i+1} \otimes \boldsymbol{L}_{i+2}\right\|} \frac{3\left\|\boldsymbol{L}_{i+1}\right\|}{\left\|\boldsymbol{L}_{i}\right\|+\left\|\boldsymbol{L}_{i+1}\right\|+\left\|\boldsymbol{L}_{i+2}\right\|}
$$

where $\boldsymbol{M} \otimes \boldsymbol{N}$ denotes the cross product of the vectors $\boldsymbol{M}$ and $\boldsymbol{N}, \Delta_{i}$ denotes the volume of the hexahedron constructed by a triple of consecutive vectors $\boldsymbol{L}_{i}, \boldsymbol{L}_{\mathrm{i}+1}, \boldsymbol{L}_{i+2}$, and $\hat{\tau}_{i}$ is computed 
by Equation (3.4) at points $\boldsymbol{P}_{2}, \boldsymbol{P}_{3}, \ldots, \boldsymbol{P}_{n-1}$, and equals zero at $\boldsymbol{P}_{0}, \boldsymbol{P}_{1}$, and $\boldsymbol{P}_{n}$. Finally, the discrete torsion at each point $\boldsymbol{P}_{i}(i=2, \ldots, n-1)$ is estimated as

$$
\tau_{i}= \begin{cases}\frac{\left|\Delta_{i}\right| \hat{\tau}_{i-1}+\left|\Delta_{i-1}\right| \hat{\tau}_{i}}{\left|\Delta_{i-1}\right|+\left|\Delta_{i}\right|}, & \Delta \Delta_{i-1}>0 \\ 0 \quad, & \Delta \Delta_{i-1} \leq 0\end{cases}
$$

When the points $\boldsymbol{P}_{i-2}, \boldsymbol{P}_{i-1}, \boldsymbol{P}_{i}$ and $\boldsymbol{P}_{i+1}$ are coplanar, $\tau_{i}=0$; Otherwise, $\tau_{i} \neq 0$. The sign and absolute value of $\tau_{i}$ determine the direction and degree of the tortility of the final interpolation curve at $\boldsymbol{P}_{i}$, respectively. Hence, the parameter correction induced by the torsion at a point can be expressed as $f_{i}=\beta\left|\tau_{i}\right|$, where the scale $\beta$ can be set as a constant or variable. Thus the parameter $t_{i}$ associating with $\boldsymbol{P}_{i}$ can be formulated as

$$
t_{i}=t_{i-1}+\left\|\boldsymbol{L}_{i}\right\|^{0.5} / D+\bar{e}_{i}+f_{i}
$$

where $\bar{e}_{i}$ is computed by Equation (3.1). For 2D points, all the discrete torsions calculated by Equation (3.5) are zero, and thus the Equation (3.6) is equivalent to Equation (3.3). Consequently, the proposed method depicted by Equation (3.6) is appropriate for both $2 \mathrm{D}$ and 3D cases.

\subsection{Criterion of interpolation precision measurement}

The knot vector and interpolation curve can be constructed after the parameter values being computed by Equation (3.6). Different parameter values usually result in different shapes of curves and the deviation values of those curves to the polyline of the data points may be different. There are many criteria to measure the divergence [16-18]. In the paper, we utilized the curvature and Euler distance-based criterion depicted in [9] to compare different methods.

\subsection{Value range for $\alpha$ and $\beta$}

Experimental results show that the optimum value of $\alpha$ has relation with the shortest side length of the triangles constructed by each triple of consecutive points, i.e. the bigger the side length is, the smaller the value of $\alpha$ will be. The value of the torsion augmented coefficient $\beta$ has relation with the points' torsions, i.e. the bigger the torsions are, the smaller the optimum value of $\beta$ will be. In this work, we recommend using 0.05 as an initial value for $\beta$.

\section{Numerical experiments and discussion}

\subsection{Numerical experiments}

We compare our approach developed in Section 3.3 with the chord length [1], centripetal $[1,4]$ and Fang's methods [9] by four examples. For the sake of justice, the curvature and Euler distance-based criterion for the deviation of the final interpolation curve from the data points is chosen as the unified comparison standard for the four methods. We choose the cubic B-spline curve as interpolation curve and the average method to calculate the knot vector $\boldsymbol{T}=\left\{\tau_{j}\right\}$. Once the parameters $\left\{t_{i}\right\}$ is get by one of the four approaches and the knot vector $\boldsymbol{T}$ is achieved, the interpolation curve will be obtained by algorithms for calculating a system of linear equations $[1,10,19]$ or by a robust progressive iteration algorithm[20,21] with a small threshold, such as $1.0 * 10^{-8}$.

Example 1. The data set contains 12 planar points, i.e. $\boldsymbol{P}_{0}=[1 ; 1], \boldsymbol{P}_{1}=[2 ; 1.2], \boldsymbol{P}_{2}=[3 ; 1]$, $\boldsymbol{P}_{3}=[4 ; 1.3], \boldsymbol{P}_{4}=[6 ; 8], \boldsymbol{P}_{5}=[7 ; 1.25], \boldsymbol{P}_{6}=[7.5 ; 2], \boldsymbol{P}_{7}=[8 ; 1.2], \boldsymbol{P}_{8}=[8.2 ;-3.2], \boldsymbol{P}_{9}=[11 ; 0.3], \boldsymbol{P}_{10}=[12 ; 4]$, $\boldsymbol{P}_{11}=[15 ;-2]$. The polyline connecting the points is shown in Fig. 4(a), and the cubic B-spline 
curves obtained by Fang's, the proposed, the centripetal and the chord length methods are shown in Fig. 4(b) to Fig. 4(e) respectively. Distance deviations computed by the four methods with different values of $\alpha$ are listed in Table 1 and the centripetal method and the chord length one are irrelative with $\alpha$. The Fang's method and the proposed one get the minimum deviations 1.512049 and 1.449913 with $\alpha=0.016$ and 0.017 , respectively. Fig. 4(e) shows that the final curve obtained by chord length method has loops and spires, which are not acceptant in CAD and CAGD domains.

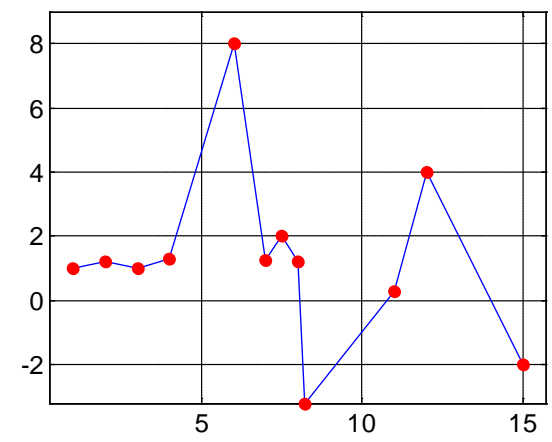

a. Data points and the polyline
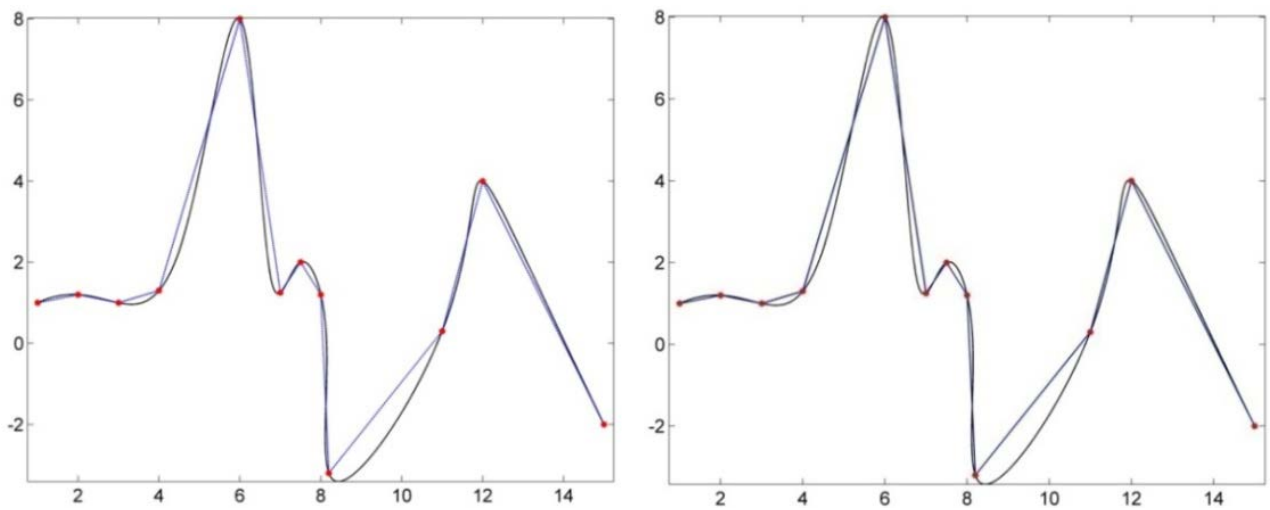

b. Fang's method

c. Proposed method
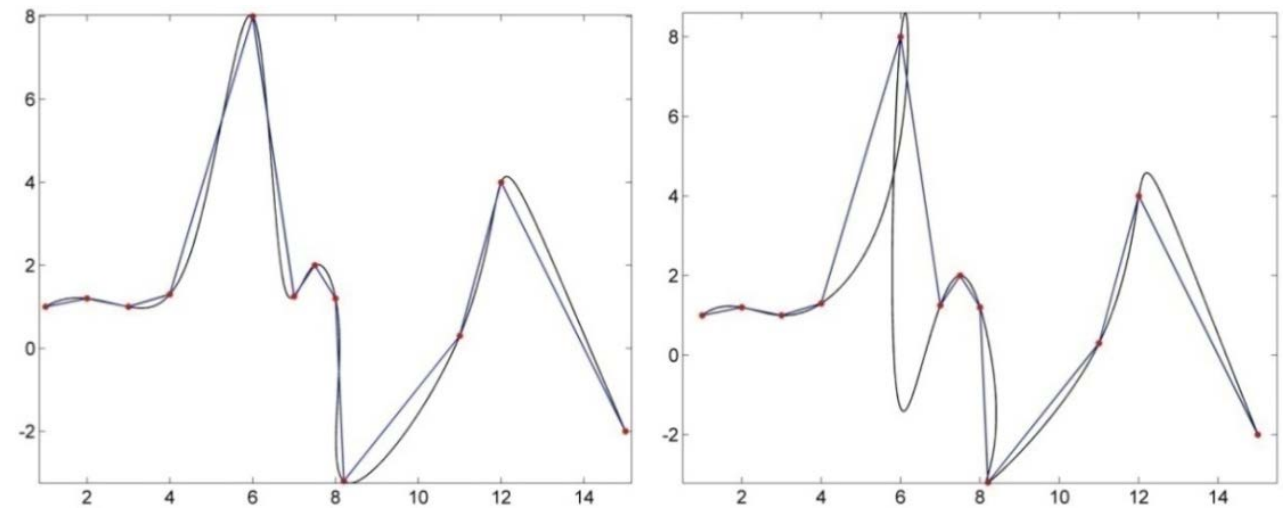

d. Centripetal method

e. Chord length method

Figure 4: Data points and interpolation curves by the four methods (Example 1)

\begin{tabular}{cccccccc}
\hline$\alpha$ & 0.014 & 0.015 & 0.016 & 0.017 & 0.018 & 0.019 & 0.020 \\
\hline Fang’s & 1.517790 & 1.513974 & 1.512049 & 1.513629 & 1.517409 & 1.522162 & 1.527457 \\
Proposed & 1.457083 & 1.452770 & 1.450377 & 1.449913 & 1.451091 & 1.453554 & 1.457285 \\
Centripetal & & & & 1.677775 & & & \\
Chord length & & & & 18.737667 & & & \\
\hline
\end{tabular}

Table 1: Distance deviation of the four methods in example 1

Example 2. The data points are sampled from a hypotrochoid curve determined by $x=100 \times((a-b) \times \cos (t)+h \times \cos ((a-b) / b \times t)), \quad y=100 \times((a-b) \times \sin (t)-h \times \sin ((a-b) / b \times t))$, where $a=1$, 
$b=3 / 4, h=9 / 13, t=[-3 \pi, 3 \pi]$ and the sampling step $=\pi / 2$. The data set contains 13 points and the polyline connected them is shown in Fig. 5(a). The cubic B-spline curves obtained by Fang's method, the proposed method, the centripetal method and the chord length method are shown in Fig. 5(b) to Fig. 5(e) respectively. Distance deviations computed by the four methods with different values of $\alpha$ are listed in Table 2. Fang's method and the proposed one get the minimum deviations 4.185482 and 4.181231 with $\alpha=0.00007$ and 0.00018 , respectively.

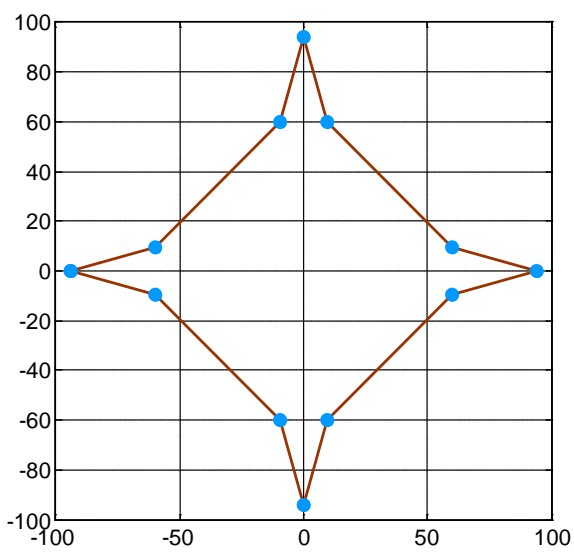

a. Data points and the polyline
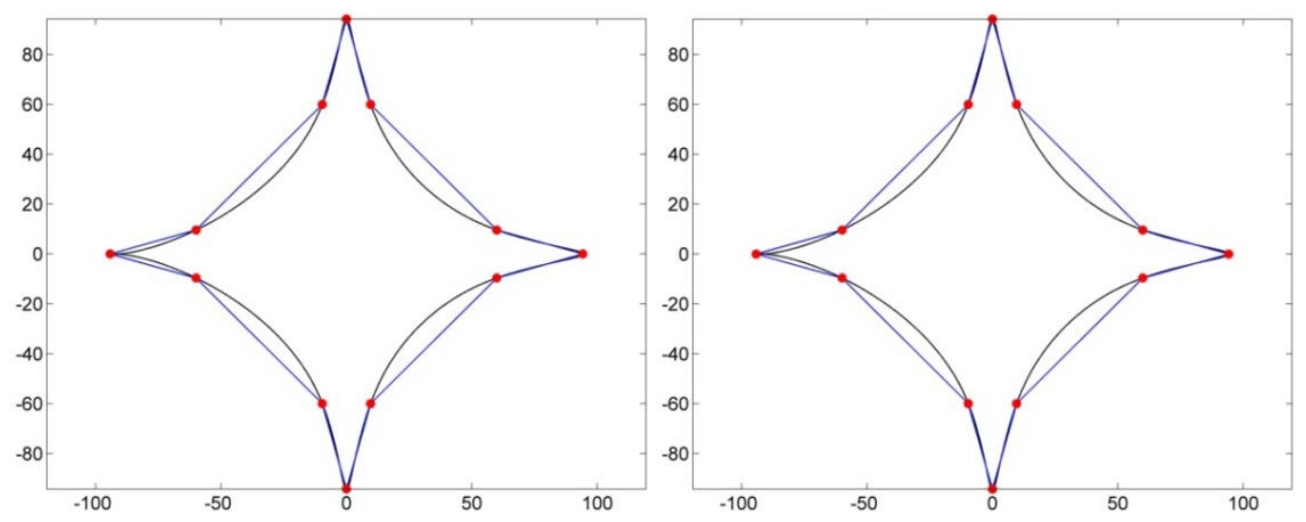

b. Fang's method

c. Proposed method
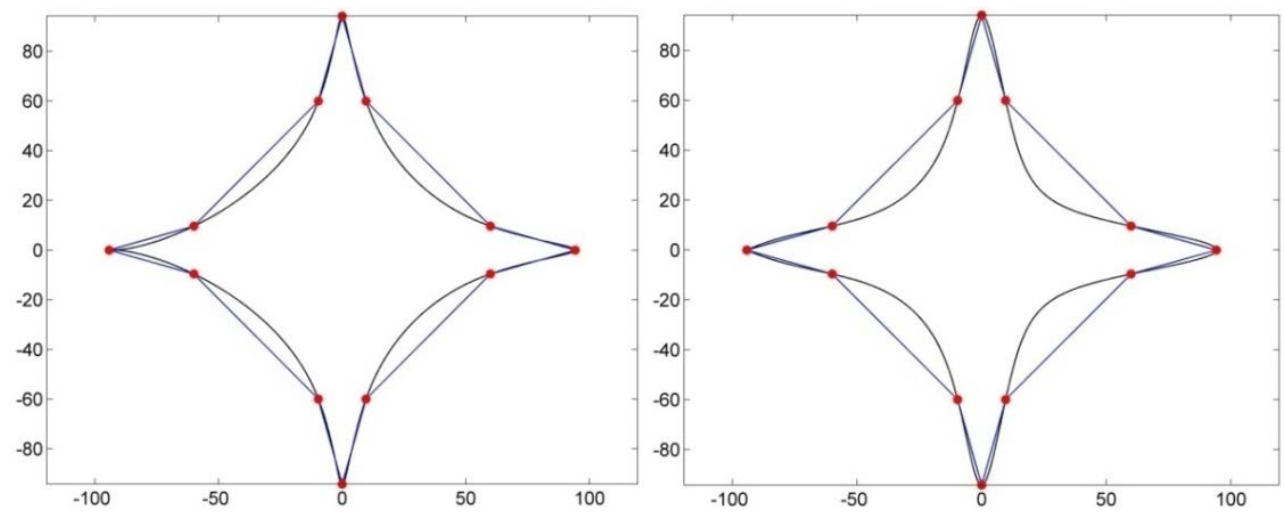

d. Centripetal method

e. Chord length method

Figure 5: Data points and interpolation curves by the four methods (Example 2)

\begin{tabular}{ccccccccc}
\hline$\alpha$ & 0.00006 & 0.00007 & 0.00008 & 0.0001 & 0.00015 & 0.00017 & 0.00018 & 0.00019 \\
\hline Fang's & 4.185522 & 4.185482 & 4.185545 & 4.185986 & 4.188297 & 4.189704 & 4.190466 & 4.191382 \\
Proposed & 4.183784 & 4.183378 & 4.183039 & 4.182450 & 4.181403 & 4.181296 & 4.181231 & 4.181261 \\
Centripetal & & & \multicolumn{7}{c}{4.187168} \\
Chord length & & & 6.720811 & & & \\
\hline
\end{tabular}

Table 2: Distance Deviation of the Four Methods in Example 2 
Example 3. The data set contains $93 \mathrm{D}$ points, i.e. $\boldsymbol{P}_{0}=[1 ;-2 ; 0], \boldsymbol{P}_{1}=[1 ; 1 ; 0], \boldsymbol{P}_{2}=[0 ; 1 ; 0]$, $\boldsymbol{P}_{3}=[0 ; 1 ; 1], \boldsymbol{P}_{4}=[0 ; 2 ; 1], \boldsymbol{P}_{5}=[1 ; 3 ;-3.5], \boldsymbol{P}_{6}=[2 ; 3 ; 1], \boldsymbol{P}_{7}=[2 ; 1.5 ; 0]$ and $\boldsymbol{P}_{8}=[3 ; 1.5 ; 0]$. The polyline connecting the points is shown in Fig. 6(a), and the cubic B-spline curves obtained by Fang's method, the proposed method, the centripetal method and the chord length method are shown in Fig. 6(b) to Fig. 6(e), respectively. Distance deviations computed by the four methods with $\beta=0$ and different values of $\alpha$ are listed in Table 3, in which, Fang's method and the proposed one get the minimum deviation 2.413632 and 2.396702 with $\alpha=0.03$, respectively. Distance deviations computed by our method with $\alpha=0.03$ and different values of $\beta$ are listed in Table 4 and our method get the minimum value 2.390160 at $\beta=0.16$.

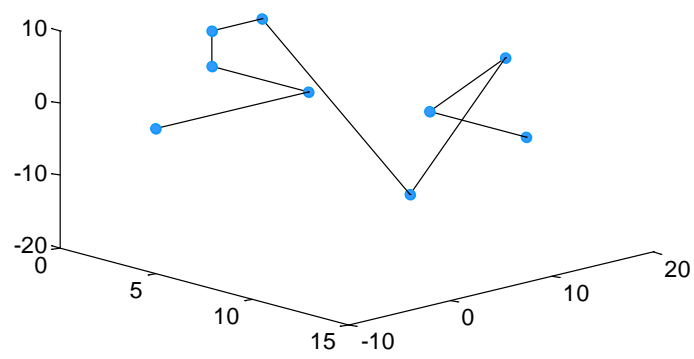

a. Data points and the polyline
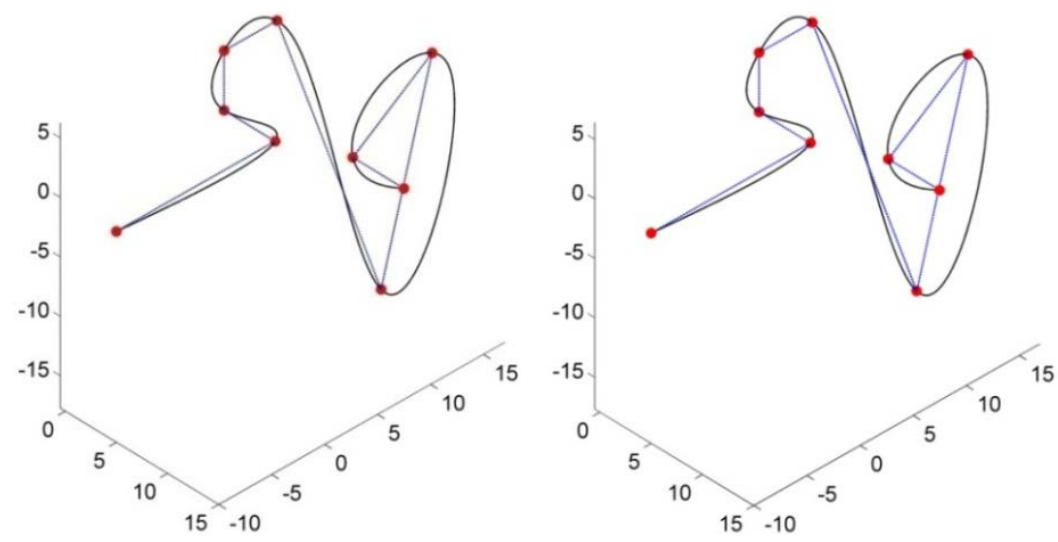

b. Fang's method

c. Proposed method
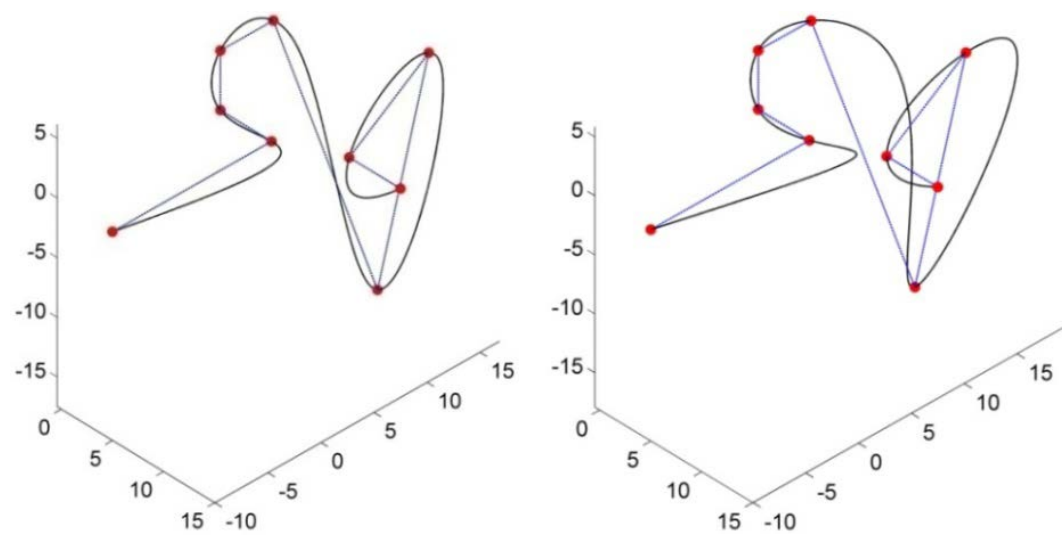

d. Centripetal method

e. Chord length method

Figure 6: Data points and interpolation curves by the four methods (Example 3)

\begin{tabular}{ccccccc}
\hline$\alpha$ & 0.01 & 0.02 & 0.03 & 0.04 & 0.05 & 0.06 \\
\hline Fang's & 2.518915 & 2.426647 & 2.413632 & 2.418041 & 2.430161 & 2.450619 \\
Proposed & 2.519913 & 2.416239 & 2.396702 & 2.397967 & 2.405062 & 2.423769 \\
Centripetal & & & \multicolumn{2}{c}{3.116156} & & \\
Chord length & & & 5.014220 & & \\
\hline
\end{tabular}


Table 3: Distance Deviation of the Four methods in Example $3(\beta=0)$

\begin{tabular}{cccccccc}
\hline$\beta$ & 0.13 & 0.14 & 0.15 & 0.16 & 0.17 & 0.18 & 0.19 \\
\hline Proposed & 2.390215 & 2.390167 & 2.390162 & 2.390160 & 2.390251 & 2.390391 & 2.390561 \\
\hline
\end{tabular}

Table 4: Distance deviation of the proposed method in example $3(\alpha=0.03)$

Example 4. The data set contains nine3D points, i.e. $\boldsymbol{P}_{0}=[20 ; 0 ; 0], \boldsymbol{P}_{1}=[\mathrm{s} ; \mathrm{s} ;-10], \boldsymbol{P}_{2}=[0 ; 20 ; 0]$, $\boldsymbol{P}_{3}=[-\mathrm{s} ; \mathrm{s} ;-10], \boldsymbol{P}_{4}=[-20 ; 0 ; 0], \boldsymbol{P}_{5}=[-\mathrm{s} ;-\mathrm{s} ;-10], \boldsymbol{P}_{6}=[0 ;-20 ; 0], \boldsymbol{P}_{7}=[\mathrm{s} ;-\mathrm{s} ;-10]$ and $\boldsymbol{P}_{8}=[20 ; 0 ; 0]$, where $s=40 \times \sqrt{2} / 2$. The polyline connecting the points is shown in Fig. 7(a), and the cubic B-spline curves obtained by Fang's method, the proposed method, the centripetal method and the chord length method are shown in Fig. 7(b) to Fig. 7(e) respectively. Distance deviations computed by the four methods with $\beta=0$ and different values of $\alpha$ are listed in Table 5 and also the centripetal and chord length methods are irrelative with $\alpha$ In Table 6, Fang's method and the proposed achieve the minimum deviation 5.017925 and 5.017953with $\alpha=0.0012$, respectively. The distance deviations computed by our method with $\alpha=0.0012$ and different values of $\beta$ are listed in Table 6 and our method is the minimum value 4.630315 with $\beta=0.0018$.

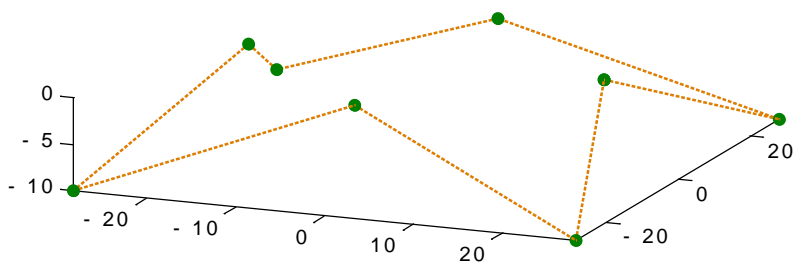

a. Data points and the polyline

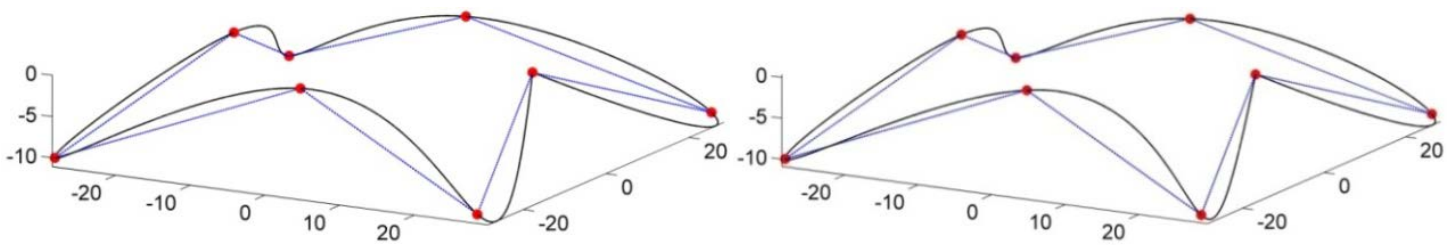

b. Fang's method

c. Proposed method

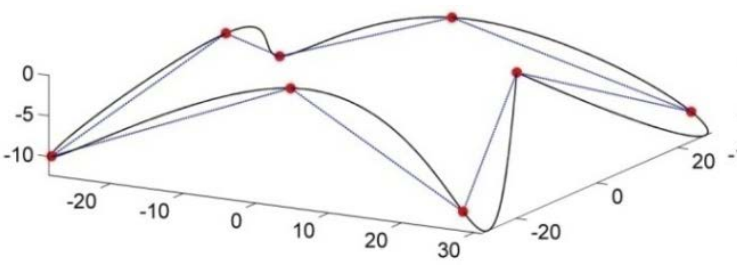

d. Centripetal method

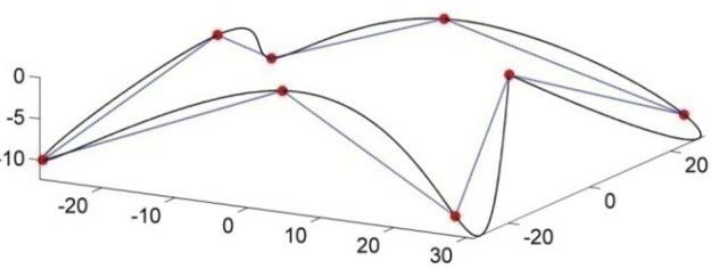

e. Chord length method

Figure 7: Data points and interpolation curves by the four methods (Example 4)

\begin{tabular}{ccccccc}
\hline$\alpha$ & 0.0008 & 0.0009 & 0.001 & 0.0011 & 0.0012 & 0.0013 \\
\hline Fang’s & 5.034880 & 5.026639 & 5.021550 & 5.018686 & 5.017925 & 5.018950 \\
Proposed & 5.034586 & 5.026297 & 5.021331 & 5.018554 & 5.017953 & 5.019288 \\
Centripetal & & & 5.286629 & & \\
Chord length & & \multicolumn{5}{c}{5.286629} \\
\hline
\end{tabular}

Table 5: Distance Deviation of the Four Methods in Example $4(\beta=0)$

\begin{tabular}{cccccccc}
\hline$\beta$ & 1.5 & 1.6 & 1.7 & 1.8 & 1.9 & 2.0 & 2.1 \\
\hline Proposed & 4.639045 & 4.633862 & 4.630822 & 4.630315 & 4.631928 & 4.635823 & 4.641646 \\
\hline
\end{tabular}

Table 6: Distance Deviation of the Proposed Method in Example $4(\alpha=0.0012)$

\subsection{Discussion}




\subsubsection{Algorithms comparison}

From Table 1 to Table 3, the minimum distance deviation of our method is smaller than that of the Fang's, centripetal and chord length methods when the discrete torsion information at each point is not taken into account. From Table 5, the minimum deviation of our algorithm with $\beta=0$ is smaller than that of the Fang's about $2.8 \times 10^{-5}$.

From Table 3 to Table 6, the proposed method can efficiently reduce the deviations when the discrete torsions are taken into account. As compared with Fang's approach, the deviation values of the proposed scheme for Examples 3 and 4 reduce about 0.023472 and 0.38761 , respectively. As compared with the centripetal and chord length methods, the proposed one possesses many advantages.

From Table1 to Table 6, the proposed method can obtain a minimum deviation value in a large range of parameter values and there is a pair of values of $\alpha$ and $\beta$ that makes the parameterization results of our method being finer than the other three ways.

\subsubsection{Future work}

There are several possibilities for the extension of our method; we list a few of them as follows:

(1) The parameters of the data points have tight relations with the knot vector $\boldsymbol{T}$ and both of them have coordinative importance for the shape of the final interpolation curve. However, the relations among parameter values $\left\{t_{i}\right\}$, the knot vector $\boldsymbol{T}$ and the arc length parameter of the final curve are not very clear and it is worthy of much more research.

(2) Another extension is to adopt different $\alpha_{i}$ and $\beta_{i}$ for each parameter interval $\Delta t_{i}$ by taking the properties of concave and convexity of the given points into account.

\section{Conclusion}

Aiming at the shortages of common parameterization methods and Fang's improved centripetal method [9], a new parameterization method, based on discrete curvature and torsion, is provided and illumined by the local differential geometric properties of a space curve at one point. Experimental results indicate that the distance deviation between the B-spline interpolation curve obtained by our method and the polyline of data points is smaller when compared with the centripetal, chord length and Fang's parameterization, thus the proposed method is more excellent for 2D and 3D data points' parameterization.

\section{References}

[1] L.A. Piegl and W. Tiller. The NURBS Book[M]. Berlin: Springer-Verlag. 364-365(1997)

[2] S.M. Shamsuddin, M.A. Ahmed, Y. Samian. NURBS skinning surface for ship hull design based on new parameterization method[J]. I.J.A.M.T. 28(9): 936-941(2006)

[3] J.F. Huang, S.L. Wan. The parametric method of Friendship hull form based on design feature [J]. Chinese Journal of Ship Research, 7(2): 79-85(2012)

[4] E. Lee. Choosing nodes in parametric curve interpolation[J]. CAD, 21(6): 363-370(1989)

[5] J. Hoschek, D. Lasser. Fundamentals of computer aided geometric design[M]. London: A.K. Peters, 1993.

[6] T.A. Foley, G.M. Nielson. Knot selection for parametric spline interpolation[C]. Mathematical methods in computer aided geometric design, Academic Press Professional: 261-272(1989)

[7] C-G. Lim. A universal parameterization in B-spline curve and surface interpolation [J]. CAGD, 16(5): 407-422(1999) 
[8] C-G. Lim. Universal parameterization in constructing smoothly-connected B-spline surfaces[J]. CAGD, 19(6): 465-478(2002)

[9] J.J. Fang, C.L. Hung. An improved parameterization method for B-spline curve and surface interpolation[J]. CAD, 45(6): 1005-1028(2013)

[10] N.M. Patrikalakis, T. Maekawa. Shape Interrogation for Computer Aided Design and Manufacturing[M]. Berlin: Springer. 35-47(2002)

[11] D. Coeurjolly, S. Miguet, T. Laure. Discrete Curvature Based on osculating circle estimation[C].Lecture notes in computer science, Heidelberg: Springer. 303-312(2001)

[12] G.H. Liu, Y.S. Wong, Y.F. Zhang, H.T. Loh. Adaptive fairing of digitized point data with discrete curvature[J]. CAD, 34(4), 309-320(2002)

[13] T. Lewiner, JD Gomes Jr., H. Lopes, M. Craizer. Curvature and torsion estimators based on parametric curve fitting[J]. C\&G, 29(5): 641-655(2005)

[14] V.P. Kong, BH Ong. Shape preserving F3 curve interpolation[J]. CAGD, 19(4): 239-256(2002)

[15] V.P. Kong, BH Ong. Shape preserving approximation by spatial cubic splines[J]. CAGD, 26(26): 888-903(2009)

[16] WP Wang, H Pottmann, Y Liu. Fitting B-spline curves to point clouds by curvature-based squared distance minimization[J]. ACM TOG, 25(2): 214-238(2006)

[17] H Park, J-H Lee. B-spline curve fitting based on adaptive curve refinement using dominant points[J]. CAD, 39(6): 439-451(2007)

[18] CM Zhang, WP Wang, JY Wang, XM Li. Local computation of curve interpolation knots with quadratic precision[J]. CAD, 45(4): 853-859(2013)

[19] S Gofuku, S Tamura, T Maekawa. Point-tangent/point-normal B-spline curve interpolation by geometric algorithms[J]. CAD, 41(6): 412-422(2009)

[20] HW Lin, HJ Bao, GJ Wang. Totally positive bases and progressive iteration approximation[J]. Computers and Mathematics with Applications, 50(3-4): 575-586(2005)

[21] LZ Lu. Weighted progressive iteration approximation and convergence analysis[J]. CAGD, 27(2): 129-137(2010) 